\title{
The RACE Asynchronous Collaboration Environment Project
}

\author{
T. Kiriyama, A. Kubota \\ Research into Artifacts, Center for Engineering \\ The University of Tokyo \\ 4-6-1 Komaba, Meguro-ku, Tokyo 153, Japan \\ Telephone +81.3.5453.5891 Fax +81.3.3467.0648 \\ kiriyama@race.u-tokyo.ac.jp,bota@race.u-tokyo.ac.jp
}

\begin{abstract}
The RACE Asynchronous Collaboration Environment Project (the "RACE" Project) is in progress at Research into Artifacts, Center for Engineering (RACE), the University of Tokyo. The objective of this project is to find knowledge representation that is useful for the process of reorganizing existing information into design knowledge. As a testbed of such knowledge representation, an asynchronous and distributed design environment is being built on the basis of the World-Wide Web.
\end{abstract}

Keywords

Synthesis, asynchronous collaboration, World-Wide Web

\section{INTRODUCTION}

Identifying requirements and constructing a concept are the most important tasks of the design process. Design concept may occur not only on a designer's desk but in a dialogue between the designer and the user, in an advice by a specialist, and in a discussion among designers. Computer support is supposed to take a different shape from that of the conventional CAD system centered around geometric modeling and analysis. Our concept of knowledge intensive $\mathrm{CAD}$ is a computer-augmented environment that supports a team of individuals to collaboratively create knowledge for design out of the shared information.

A key to materialize a knowledge intensive design environment is, we believe, the representation of design knowledge for synthesis. By design knowledge, we refer to information about findings and experiences in the past, which is associated to the current design goal. Our hypothesis here is that in contrast with knowledge for analysis that is usually represented in the style of classification, knowledge for synthesis rather should be represented in the style of association among a collection of information. Figure 1 compares knowledge representations for analysis and synthesis. In the process of synthesis, a collection of information is incrementally organized into a consistent explanation of the relationship between a set of requirements and a solution. Knowledge representation gives a basis on which information is collected and structured. 

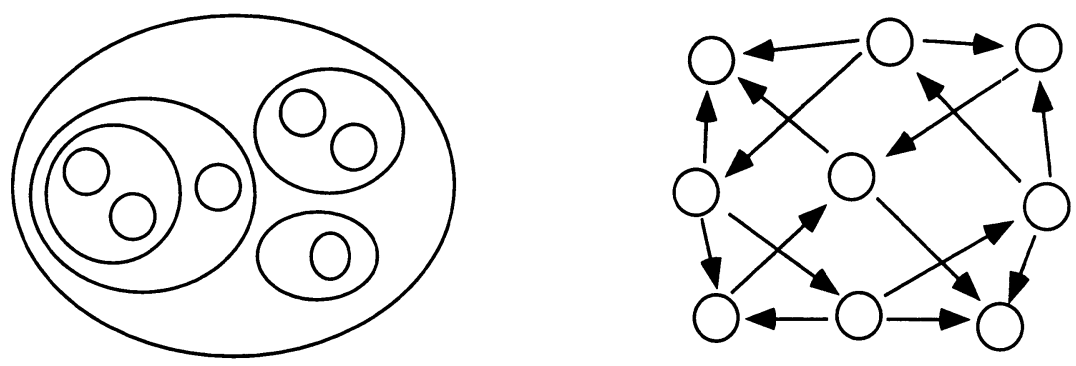

Figure 1 Knowledge representation for analysis in the left and synthesis in the right.

The view of knowledge for synthesis as associations among pieces of information can be extended to the situation of collaborative design. Members of the team provides with suggestions and explanations in various perspectives, which are integrated to be a consistent body of knowledge about the design solution. To study knowledge representation that is useful for collaborative design, we concentrate on the asynchronous mode of collaboration. As Ellis argues (Ellis 1991), software for supporting cooperative work can be categorized into modes of synchronous or asynchronous in terms of time and centralized or distributed in terms of space (Table 1). Video conferencing, for instance, supports synchronous and distributed collaboration. Since we are concerned about a team of individuals from different fields working together, we are interested in the distributed mode of collaboration. Besides, from a research point of view, an advantage of asynchronous collaboration is that exchanged information is explicitly represented. Synchronous communication such as a face-to-face meeting and a video conferencing conveys implicit information that is hard to capture. To study knowledge representation, therefore, we look at the asynchronous collaboration mode.

Table 1 Collaboration modes.

\begin{tabular}{|c|c|c|}
\hline space & centralized & distributed \\
\hline synchronous & $\begin{array}{c}\text { face-to-face } \\
\text { meeting }\end{array}$ & $\begin{array}{c}\text { video } \\
\text { conferencing }\end{array}$ \\
\hline asynchronous & $\begin{array}{c}\text { document } \\
\text { circulation }\end{array}$ & $\begin{array}{c}\text { the "RACE" } \\
\text { project }\end{array}$ \\
\hline
\end{tabular}

As an underlying technology to explore the knowledge representation for synthesis, we use the WWW (World-Wide Web). As the WWW is characterized as an embodiment of human knowledge (WWW Consortium 1992), it has grown to a huge collection of information. The idea of organizing a collection of information for synthesis fits well to the growth of links in the Web space. And the feature of the Web that everyone can provide information spontaneously is useful for a distributed design environment.

In the field of groupware (Marca 1992, Palmer 1994) and more recently in the WWW community (WWW Consortium 1995), there have been developed a variety of software to 
support collaborative work. There is, however, not much research done yet in how shared resources are transformed into useful knowledge. We need a closer look at this process to find appropriate knowledge representation.

The objective of the RACE Asynchronous Collaboration Environment project (the "RACE" project) is to find knowledge representation that is useful for the synthetic process of reorganizing existing information into design knowledge. As a testbed to explore such knowledge representation, an asynchronous, distributed collaboration environment is being built on the WWW. In the rest of this Chapter, we outline the focused areas of the "RACE" project.

\section{OVERVIEW OF THE "RACE" PROJECT}

The evolution of technologies follows a common pattern. We can distinguish three phases of a technology life-cycle, i.e., pre-competitive, competitive, and post-competitive phases. In the pre-competitive phase, a basic technology spawns out of research. Then the technology is applied to new products in the competitive phase, in which the use of technology is learned. When the technology is matured, it comes to the post-competitive stage in which knowledge about the technology becomes public. The technology as a whole evolves along the iteration of the three phases.

In the transitions of three phases, we can observe the transformation of information. In the transition from the post-competitive phase to the pre-competitive phase, knowledge about existing artifacts is used for the creation of a new design. In the transition from the precompetitive phase to the competitive phase, knowledge about combination of technologies into an integrated product is accumulated. In the transition from competitive to post-competitive phases, knowledge about the user of a technology found through the competitive phase is retrospectively formalized. Figure 2 depicts reorganization of knowledge along the transitions in the three phases.

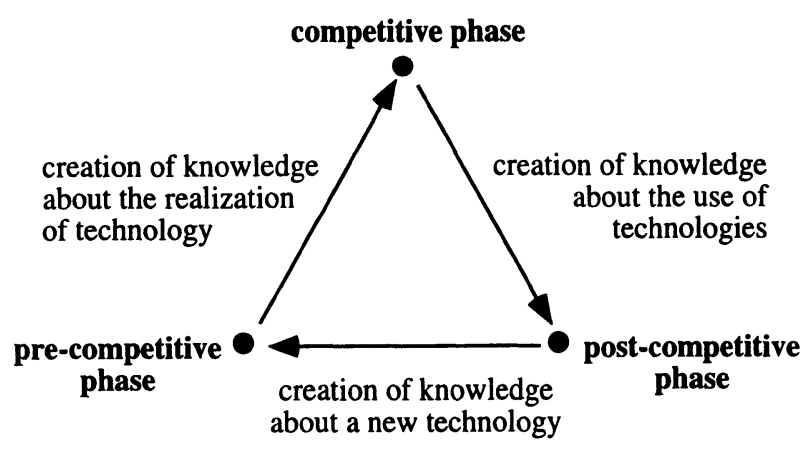

Figure 2 Transformation of knowledge in the transitions of technological phases.

We look at these phase transitions in the "RACE" project, since reorganization of knowledge is distinct. To examine knowledge transformation from the post-competitive to pre-competitive phases, we are developing Mental World Browser and Physical World Transmitter. To see the transition from the pre-competitive to competitive phases, we are observing a design process of a micro mechanism. For the transition from the competitive to post-competitive phases, we are building Green Browser. The "RACE" project comprises of the tools developed in these subprograms. 


\section{MENTAL WORLD BROWSER AND PHYSICAL WORLD TRANSMITTER}

Iterative prototyping is the central activity throughout a design process. The Mental World Browser is meant for supporting prototyping in a conceptual world, in the pre-competitive phase. The Physical World Transmitter is to be used for prototyping by manipulating shapes in a physical world (Kubota 1996).

MWB-2, running on UNIX workstations, helps the abductive formation of a concept from the huge amounts of materials on the WWW. Using an MWB-2 window as a hypothesis work space, the user can freely configure objects including keywords and icons, which are linked to WWW resources. By hitting an object, MWB-2 will direct the browser to go to the specified a WWW resource. Working with the MWB-2, the user cultivates a hypothetical concept in a stepby-step fashion.

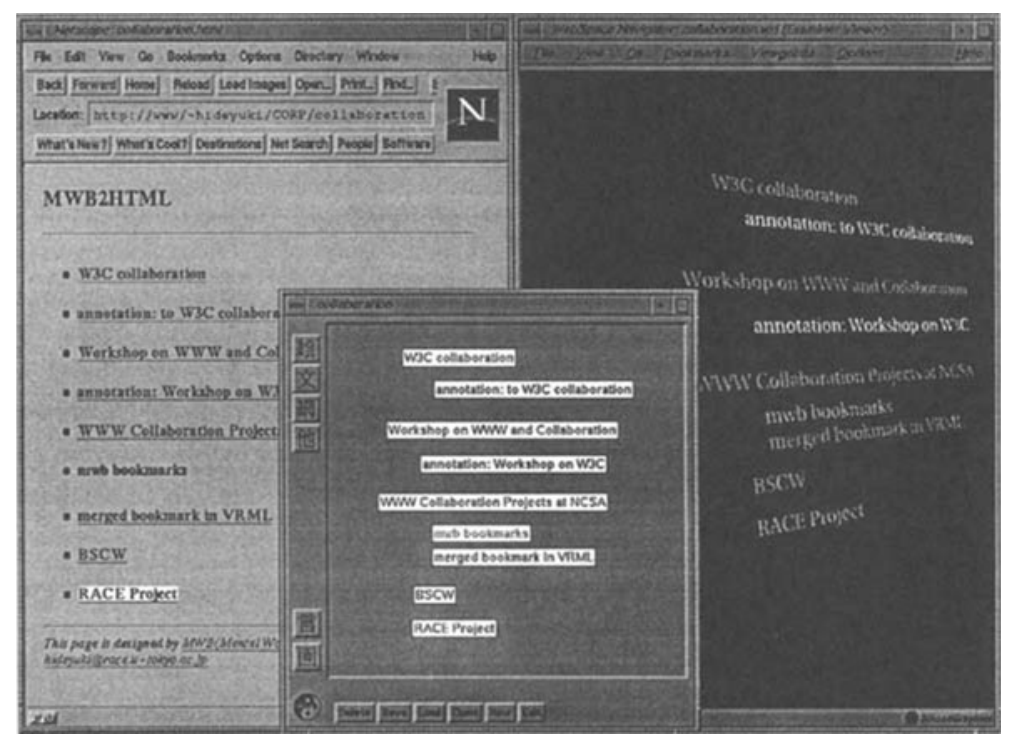

Figure 3 MWB-2, MWB2HTML, and MWB2VRML.

The MWB-2 has been extended so that it can export information from the personal field to the public field. MWB2HTML and MWB2VRML have been developed to export data. Figure 3 depicts HTML and VRML documents, exported from an MWB-2 window. To utilize heterogeneous nature of the WWW for supporting collaboration of specialists of different fields, we have developed another tool called MAC-MWB. A Finder window on the Apple Macintosh to the WWW space. As well, we have written Perl scripts called "addwrl/addinline" to merge multiple hypothesis spaces using the WWWInline node of VRML.

The Physical World Transmitter (PWT) in Figure 4 is a combination of a three-dimensional laser scanner as the input device and a stereo lithography system as the output device. It is used to transfer and reproduce shapes across the Internet. It is an alternative way of traditional prototyping using clay models and sketches. MWB and PWT complement each other in prototyping the design concept. 


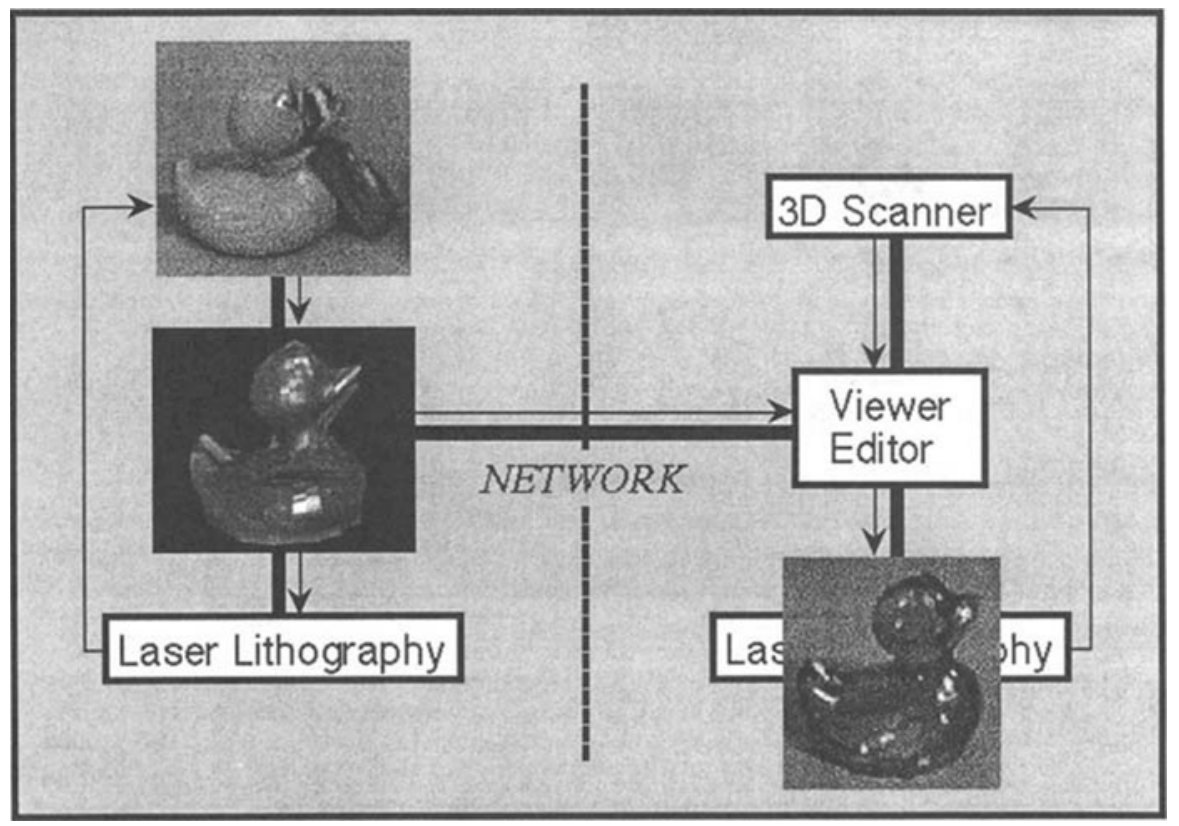

Figure 4 Physical World Transmitter.

\section{DESIGN OF MICRO ACCELEROMETER}

Design of micromehcanism devices needs various kind of engineering knowledge about fabrication, materials, dynamics, electronics, and computer analysis. To examine the process of integration of such knowledge in the competitive phase, we have been working on the design and manufacturing of a micro accelerometer (Kiriyama 1996). In this design, we try to learn what kind of information sharing is useful to support iterative prototyping toward materialization. The members of the design team are located at the University of Tokyo and Cambridge University. Members at Cambridge University are specialized in micro fabrication techniques and design process analysis, and members at the University of Tokyo are responsible for computer analysis, the network environment, and micro assembly technology.

A fabrication test of the micro accelerometer is shown in Figure 5. The technology of focused ion-beam cutting is used to make a fine cutting in the bridge between the proof mass and its counter part. This configuration, however, does not produce an enough amount of deflection for the required resolution. To increase the mass, we decided to integrate the technology of micro assembly to bond a proof-mass on the cantilever. 


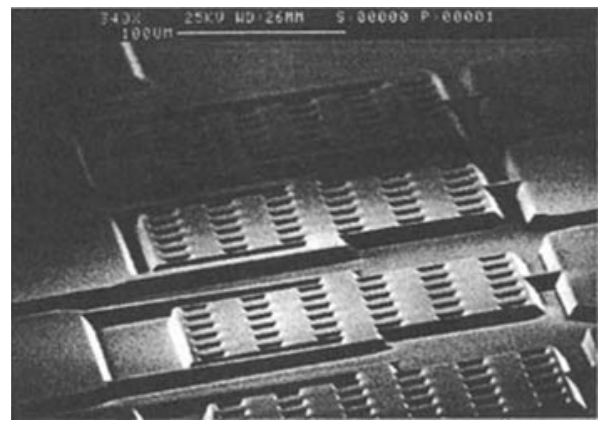

Figure 5 Micro accelerometer.

For finite element analysis of the dynamic and electrostatic behavior of micromechanisms, a CAE system has been developed by Yoshimura and his colleges (Lee 1995). The system automatically generates finite elements and appropriate analysis conditions, which is then passed on to an analysis package. A self-explanatory interface to the WWW for submitting a finite element analysis and receiving the result has been created using FORM and CGI scripts. The interface allows the user to submit an analysis over the network and receive the result. Values of resonant frequencies in the result are presented in HTML, and geometric models of vibration modes are in VRML. In the interface shown in Figure 6, the user is asked to specify dimensions and material properties.

For information sharing we have used HyperNews, which allows members to post articles in HTML. We exchanged information by e-mails and posted relatively fixed results to the HyperNews. Another useful way of the HyperNews is to store input models and output results of FEM analysis. It allows all members of the team to review the results of analysis.
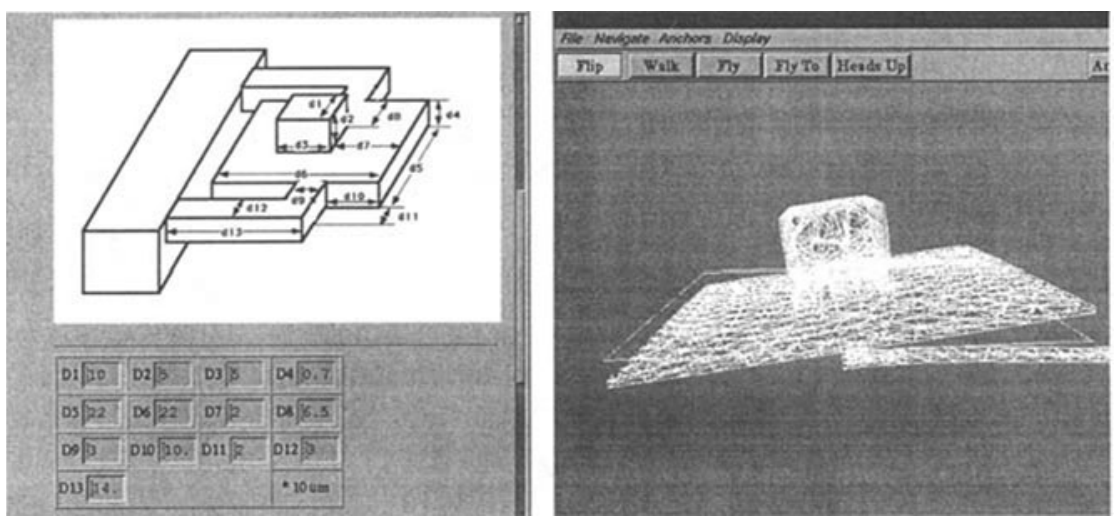

Figure 6 Interface for finite element analysis. 


\section{GREEN BROWSER}

Decision making over conflicting requirements is an important activity of synthesis. The competitive phase of technology is full of such cases, and it is an important source of information for post-competitive formalization of design knowledge. The Green Browser (Kurakawa 1996) is developed to serve as a tool to model trade-off relationships between requirements and decisions made over them.

As illustrated in Figure 7, the green browser consists of a strategy model, a process model, and object models. The strategy model is the central model of the Green Browser, which represents requirements for product. The process model represents a product's life cycle, and the object models describe the product's attributes. In the strategy model, requirements for the product are linked by their relationships of positive, negative, and trade-off. Each of requirements and relationships is associated with its description.

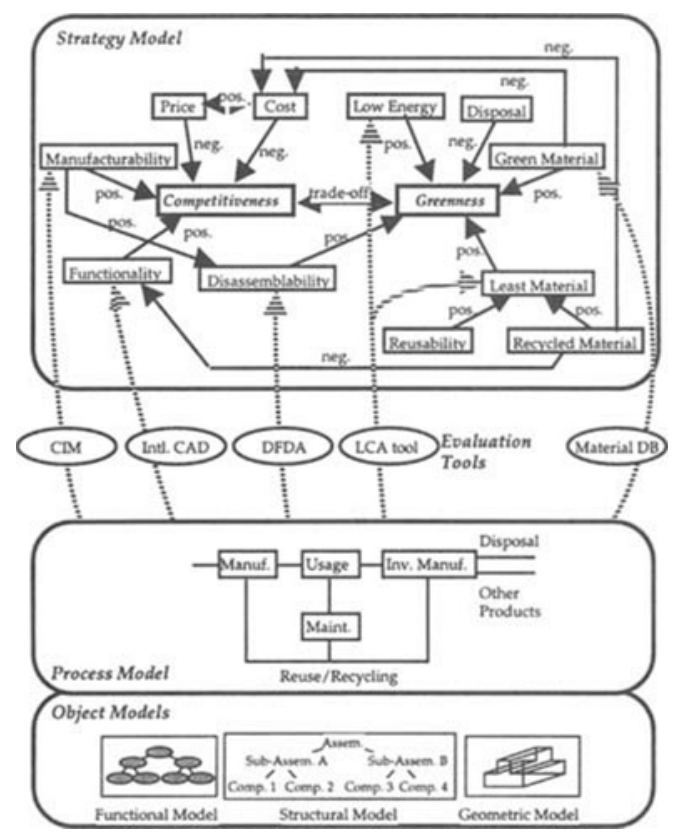

Figure 7 Model of the Green Browser.

The green browser is built on top of MOO (MUD (Multi User Domain) Object-Oriented). MOO allows multiple users read and write the data. A strategy model is stored in the data space of MOO. The MOO we are using has been augmented with an interface to the WWW (Meyer 1994). A node in the strategy model can be shown as an HTML document and a link is shown as links between the documents. The network structure of the strategy model can be mapped into a three dimensional space and displayed using VRML browsers. By clicking on a node or a link, the user can read the document on an HTML browser. Figure 8 depicts a node of the strategy model. 


\section{CONCLUSIONS}

In this paper we discussed the concept of the "RACE" project and its components. The objective of the project is to understand knowledge representation that is suitable for the synthetic process of reorganizing information into design knowledge. To explore it, we focus on the technological transitions between pre-competitive, competitive, and post-competitive phases.

Currently, MWB-2 and PWT are used to design a space for thinking and discussion called Abduction Machine 1 in collaboration with artists at Wood Studio of the Tokyo National University of Fine Arts and Music. The micro mechanism project is in the status of experimental fabrication. The Green Browser is under study of effective presentation of the strategy model. Future work common among these tools should include quantitative analysis of the manipulation of information, as it has been tried on the media of distributed collaboration in (Reddy 1995).

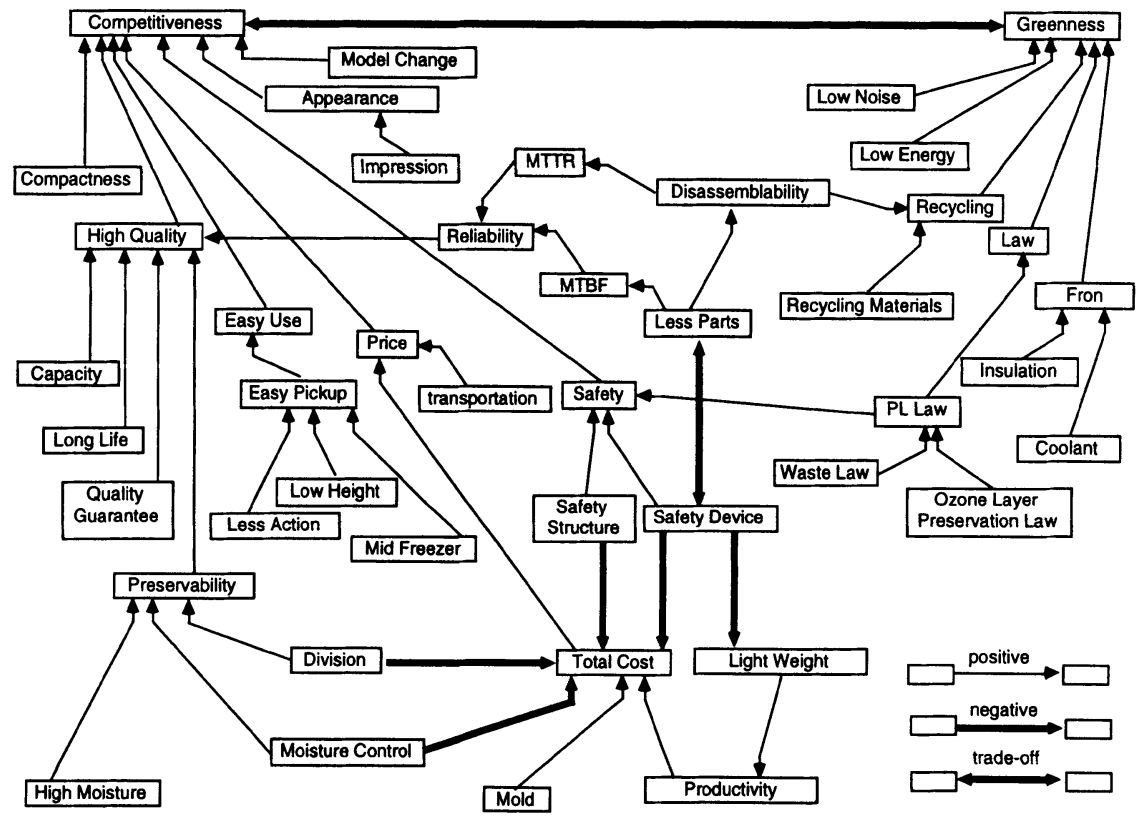

Figure 8 The strategy model.

\section{ACKNOWLEDGMENT}

The authors are grateful to Koji Ando, Hideyuki Ando, Motohiro Asai, Maki Itoh, Atsushi Kubota, Kei Kurakawa, Ichiro Nagasaka, Masanori Oosaki, Makoto Tabuchi, Atsushi Yamagishi, and Hitoshi Yamauchi for their contributions to the "RACE" project. 


\section{REFERENCES}

Ellis, C.A., Gibbs, S.J., Rein, G.L (1991) Groupware: Some Issues and Experiences, Communications of the ACM. Vol. 34, No.1, 38-58.

Kiriyama, T., Nakajima, N., Yoshimura, S., Burgess, S.C., Moore, D.F., Shibaike, N. (1996) A Conceptual Design Environment for Micromechanisms, Proceedings of the European Design \& Test Conference, IEEE, 448-453.

Kubota, A. (1996) Abduction Machine Project, Proceedings of Second International Symposium on Creativity and Cognition, 77-84.

Kurakawa, K., Kiriyama, T., Baba, Y., Kobayashi, H., Umeda, Y., Tomiyama, T. (1996) The Green Browser: An Information Sharing Tool for Product Life Cycle Design, Proceedings of ASME Design Technical Engineering Conference and Computers in Engineering Conference, (to appear).

Lee, J.S., Yoshimura, S., Yagawa, G., Shibaike, N. (1995) A CAE System for Micromachines: Its Application to Electrostatic Micro Wobble Actuator, Sensors and Actuators A 50, Elsevier, 209-221.

Marca, D., Bock G. (1992) Groupware: Software for Computer-Supported Cooperative Work, IEEE.

Meyer T., Blair D., Hader, S. (1994) A MOO-Based Collaborative Hypermedia System for WWW.

Palmer, J.D., Fields, N.A. (1994) Computer-Supported Cooperative Work, IEEE Computer, May 1994, 15-17.

Reddy, J.M., Chan, B., Finger, S. (1995) Patterns in Design Discourse: A Case Study, Proceedigns of the First IFIP WG 5.2 Workshop on Knowledge Intensive CAD, 321343.

WWW Consortium (1995) Proceedings of Workshop on WWW and Collaboration.

WWW Consortium (1992) About the World Wide Web, http://www.w3.org/pub/Www/WwW/

\section{BIOGRAPHY}

Dr. Takashi Kiriyama graduated from Department of Precision Machinery Engineering, Faculty of Engineering, the University of Tokyo in 1986. He obtained his Doctor's degree in Engineering from the University of Tokyo in 1991. In 1992 he joined Research into Artifacts, Center for Engineering (RACE), the University of Tokyo as lecturer, and has been associate professor since 1995. His research interest includes artifactural engineering, design theory, collaboration in design, and qualitative physics.

Dr. Akihiro Kubota has been Associate Professor of Research into Artifacts, Center for Engineering (RACE), the University of Tokyo, since 1992. He received his doctor's degree in engineering (fluid dynamics) from the University of Tokyo in 1989. After obtaining his Dr. Eng., he worked at the Department of Naval Architecture and Ocean Engineering, the University of Tokyo until 1992. His research interest includes artifactural engineering, collaboration among experts from different fields, architecture and landscape design of knowledge, and the Internet technologies. 